\title{
Circuit
}

Musiques contemporaines

\section{Improviser ou interpréter Theatre Piece?}

Entrevue avec Malcolm Goldstein

To improvise or perform Theatre Piece?

Interview with Malcolm Goldstein

\section{Johanne Rivest}

Volume 8, numéro 2, 1997

Québecage

URI : https://id.erudit.org/iderudit/902206ar

DOI : https://doi.org/10.7202/902206ar

Aller au sommaire du numéro

Éditeur(s)

Les Presses de l'Université de Montréal

ISSN

1183-1693 (imprimé)

1488-9692 (numérique)

Découvrir la revue

Citer ce document

Rivest, J. (1997). Improviser ou interpréter Theatre Piece? Entrevue avec Malcolm Goldstein. Circuit, 8(2), 53-60. https://doi.org/10.7202/902206ar
Résumé de l'article

Rendant compte de son expérience d'interprète de pièces de Cage, le violoniste analyse minutieusement la part de l'improvisation et de la création dans leur exécution, il définit ce que veut vraiment dire « interpréter » Cage, et montre comment l'improvisateur peut se débarrasser des clichés. 


\title{
Improviser ou interpréter Theatre Piece?
}

\section{Entrevue avec Malcolm Goldstein}

\author{
Johanne Rivest
}

J'ai rencontré le compositeur, improvisateur et violoniste Malcolm Goldstein en février 1995, alors que je travaillais à ma thèse de doctorat sur John Cage. Je voulais mieux comprendre comment un interprète qui a l'habitude de l'improvisation aborde le matériau cagien, en espérant saisir davantage la portée de cette cuvre si particulière. Malcolm Goldstein a une longue feville de route concernant l'interprétation des pièces de Cage. Depuis les années soixante, lors de collaborations avec John Cage, et avec l'ensemble Tone Roads de New York, qu'il avait cofondé et dont il était codirecteur, puis à Dartmouth, Toronto et Montréal, entre autres, il a joué, dirigé, organisé ou participé à de nombreuses représentations de plusieurs des pièces de Cage, surtout indéterminées, dont le Concert for Piano and Orchestra (1957-1958), Theatre Piece (1960), Ryoanii (1983-1985) et Eight Whiskus, solos for violin (1985), composée pour lui. En tant que directeur du Hessischer Rundfunk Ensemble für Neve Musik à Francfort, il a participé au festival-anniversaire de Cage "Anarchic Harmony » en 1992.

Johanne Rivest: Vous avez exécuté Theatre Piece, de John Cage, avec un ensemble, hier, à l'Espace Tangente à Montréal "I). Comment pourriez-vous définir votre relation à cette œuvre en tant qu'interprète?

Malcolm Goldstein : Je crois qu'il y a toujours, dans le parcours de Cage, l'obligation de la clarté, de comment décrire à l'interprète ce qui est nécessaire, mais, dans ce cas, il a laissé certains éléments de confusion. Presque toutes les partitions de John Cage pendant les années soixante et soixante-dix comportent d'énormes ambiguités. Dans Theatre Piece, par exemple, il y a des zones entières où l'on doit prendre des décisions. De la part de Cage, c'est une tentative de rendre le résultat moins contrôlable afin de créer plus d'indétermination. Je ne sais vraiment pas si c'est intentionnel ou non, mais, selon ce que je connais de John, je pense que c'est intentionnel. II était attiré par une façon de penser anarchiste, sur le plan politique et musical. La clarté de ses directives ne se transforme pas en totalitarisme - comme dans Theatre Piece, où chaque personne confectionne sa propre partie et où le chef n'est pas un
(1) Parmi les pièces au programme du concert du 26 février 1995 à l'Espace Tangente figurait Theatre Piece (1960), de John Cage. Les interprètes provenaient de diverses disciplines artistiques dont la danse, la musique et la littérature. II s'agissait de Claude Cossette et Lynn Snelling, danseuses, Nicholas Caloia, contrebasse, Malcolm Goldstein, violon, et D. Kimm, écrivain. 
Figure 1: Theatre Piece, directives

THEATRE PIECE

The large numbers within brackets refer to a gamut of 20 nouns and/or verbs chosen by the performer who writes each on a card. These cards are placed (preferably face-down) so that knowing the number of each is facilitated (e.g. 5 columns of 4 or 4 of 5 ).

The large numbers are sometimes followed by smaller ones marked plus or minus. Plus means the introduction of new elements into the gamut (from a shuffled deck placed face down) at any points in the gamut and the renoval of the old ones to a reserved deck. This latter is used whenever the sign minus appears. This plus and minus operation is only done after having determined the nature of the large nurjer it accompanies.

The brackets refer to time*which may be measured by any of the rulers provided or by others made by the performer. Rulers may be changed at any points. The performer is to prepare a 30 minute program of action using as much or as little of the material provided (horizontally and vertically) as he wishes. If he requires more material horizontally he may request that it be composed.

If questions arise as to what is to be done, 4 may be asked, providing they are posed in such a way that a number or numbers $(1-20)$ will provide an answer. The numbers in the column below each bracket give these answers. $X$ is no answer (performer's free choice). None of the answers given may be used in response to more than a single question.

Pages may be performed in any order. If one extends beyond the vertical lines, an overlap (or an anticipation or extension) is indicated.

Each performer is who he is (e.g. performing musician, dancer, singer), but he is also performing a piece of theatrical music. Music is here understood to mean the production of sounds. Thus a performer's decision as to what he is to do will often be determined by whether he thus makes a sound.

A performer may include other performers in his gamut (as nouns).

For the purposes of performance, a performer may memorize his part, or may read it from carcs which he carries about or which he places at useful points on the stage. He may time his actions by reference to a stop-watch which he carries or by reference to clocks placed on the stage.

There is no conductor or director. A rehearsal will have the purpose of removing physically dangerous obstacles that may arise due to the unpredictability involved.

The lighting will be general. Alterations of this may be included in a performer's gamut (as verbs).

* These time-brackets are the time within which an action may be made. Any amount of the bracket may be used. Preparation for the action may be made at any time (outside or within the bracket); and any necessary and relevant actions following are also free with respect to time.

J.C. Stony Point, N.Y. January 1960

Copyright (C) 1977 by Henmar Press, Inc., 373 Park Ave.S., New York, NY 
Figure 2 : Theatre Piece, extrait de la partie VIII

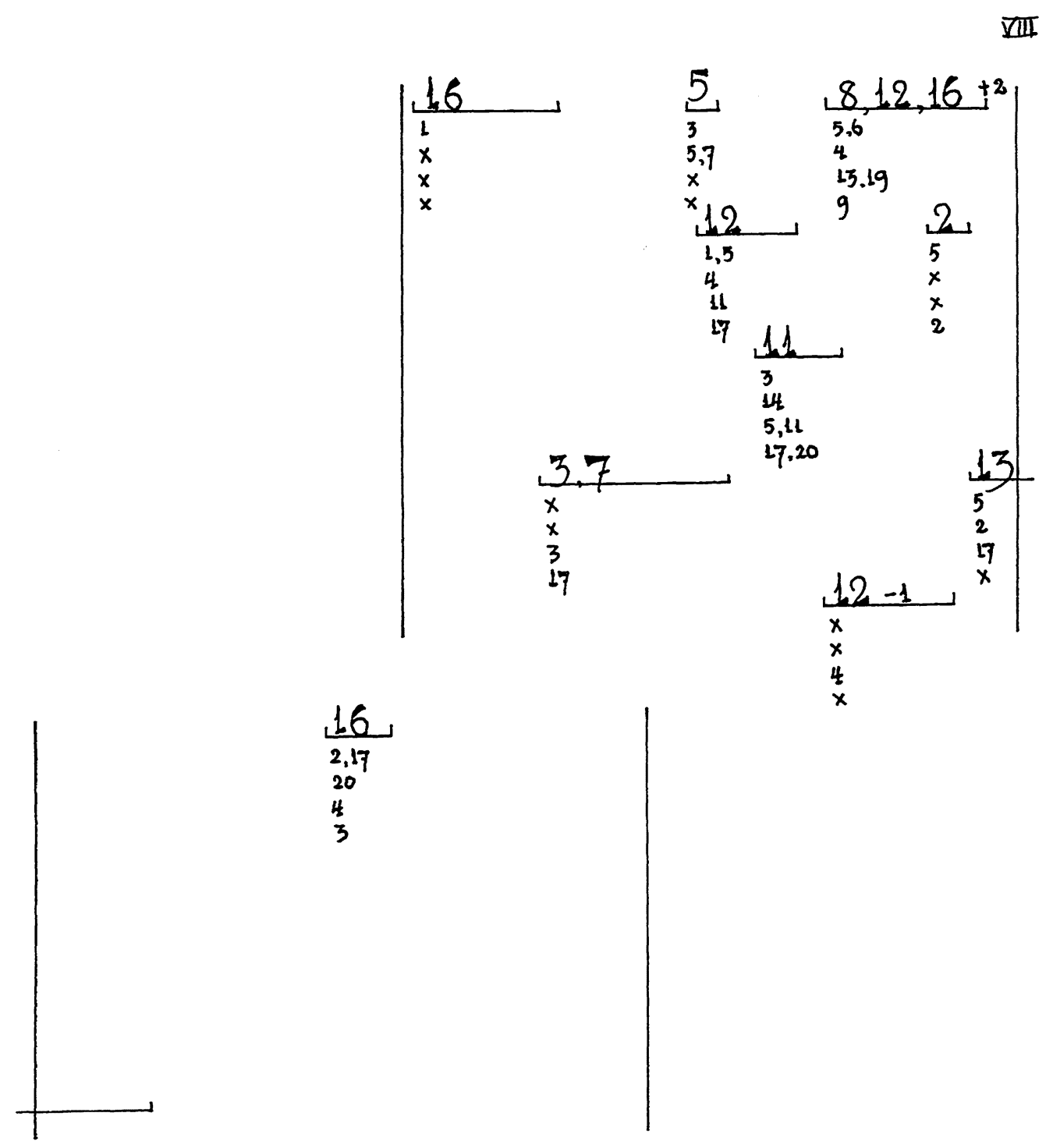

Henmar Press, (O) 1977. Reproduction autorisée par C. F. Peters. 
Chef, mais plutôt quelqu'un qui explique simplement les choses et qui fait ensuite confiance à chacun.

J.R. : Mais quelquefois la situation dans Theatre Piece semble très difficile, car si les cartes sélectionnées arbitrairement par les chiffres de la partition impliquent des activités qui ne vont pas ensemble pour un participant donné, il est alors impossible de les exécuter!

M.G. : Rien n'est impossible, pour commencer; c'est ce qu'on découvre ! Car alors on se demande: "Comment puis-je mettre le tout ensemble? » Ce qui me passionne dans ces partitions est que je n'improviserais jamais de cette manière par moi-même. À un moment donné, pendant l'exécution de Theatre Piece, je devais jover (à cause du choix initial des mots que j'avais inscrits sur les cartes) les Eight Whiskus de Cage (jover en fait de sa musique dans sa musique !), interpréter Ornette Coleman, de la musique de violoneux et je devais "disperser" le tout d'une manière ou d'une autre. Il fallait en quelque sorte trouver un moyen d'accomplir tout cela et alors j'ai vraiment dû pratiquer, pour faire un collage qui unirait les éléments entre eux. En plus de jover du violon, je pouvais chanter ou siffler certaines parties et, par là, découvrir de nouveaux modes d'exécution. Ce qui est excitant pour moi au sujet de cette musique, est que je dois choisir comment la rendre possible. De plus, j'aime à discuter certaines choses avec les interprètes, comme les questionner sur ce qu'ils peuvent faire pendant les silences et les accalmies, afin de leur permettre de s'apercevoir que tout ce qui arrive sur la scène fait partie de la représentation. C'est ce type de conscience intensifiée de ce que l'on fait qui est réellement expérimenté par l'auditoire. Silences et accalmies peuvent être vivants, simplement par notre présence.

J.R. : Croyez-vous qu'il est plus exigeant pour un interprète de jover cette sorte de musique que celle qui est entièrement écrite?

M.G. : Il s'agit de différents types d'exigences. Dans la musique où tout est écrit, la plupart des interprètes veulent pratiquer minutieusement toutes les notes. Par ailleurs, la compréhension de la conception musicale fait partie intégrante de l'exécution de la musique dont nous parlons : ce n'est pas qu'une question de notes. Les techniques pour la réaliser ne sont pas apprises à l'avance par des études à l'université ou au conservatoire. Ainsi, on y développe de nouveaux outils d'exécution, mais il faut également en comprendre la portée, trouver comment cela s'intègre à la musique. Cela renvoie l'interprète à la manière de penser propre à l'époque de Bach, où un musicien était un musicien complet. Ce genre de musique place davantage l'interprète en situation de participation au "faire " de la musique. La page écrite n'est plus alors qu'un ensemble d'activités, et l'interprète doit en comprendre le cadre, de même que l'esthétique. Par conséquent, les difficultés sont différentes. 
J.R. : Mais il y a toujours un compositeur dans ce cas, ef quelle est la différence entre l'improvisation et l'interprétation de cette musique?

M.G. : Lorsque j'ai donné un programme à Francfort ${ }^{(2)}$, je référais aux œuvres en tant que " compositions - improvisations structurées " (structured improvisation - compositions); maintenant, i'emploie l'expression « formes ouvertes". La structure peut en être rigoureuse ou très ouverte. Même si le compositeur donne le cadre de référence, il subsiste toujours un certain questionnement sur ce que I'on fait concrètement dans l'exécution musicale et alors, pour moi, l'improvisation intervient automatiquement. Cage était très critique au sujet de l'improvisation, mais en fait il était un improvisateur fantastique, comme lorsqu'il récite ses poèmes, par exemple Empty Words. II avait raison de croire, en ce sens, que l'une des difficultés de l'improvisation réside dans le fait qu'une personne finit par être tellement imprégnée de ses propres habitudes qu'elle n'improvise pas véritablement. John Cage se souciait de transcender les habitudes. Une improvisation par laquelle on cherche à démontrer combien on est fantastique n'est pas intéressante. À mon avis, l'accent en improvisation ne doit pas être mis sur l'ego, mais sur le son. La musique de Cage est formidable parce qu'elle nous place dans des situations inusitées où nous devons découvrir de nouveaux moyens. Et cela, pour moi, est également à la racine de l'improvisation. Comme dans l'exécution hier soir de Theatre Piece, l'une de mes cartes disait « imiter ». Eh bien, qui vais-je regarder? Je peux regarder l'auditoire et l'imiter. Et comment vais-je imiter ? C'est de l'improvisation pure! De plus, les répétitions sont importantes selon moi, afin que les musiciens éliminent leurs habitudes à force de jouer cette musique. Je pense que ce qui arrive avec nos habitudes, c'est qu'elles se forment si nous ne répétons la pièce qu'une ou deux fois. Mais si on recommence 4, 5, 6 ou 7 fois, alors nos habitudes disparaissent, tout simplement. À ce moment-là on devient " clair ", on commence à sentir la limpidité de la musique; on entre dans le son de l'ensemble et on ne réfléchit pas sur ce qu'il serait correct ou joli de faire; le son nous dit quoi faire.

Alors, selon moi, le principe d'être dans le moment - John aimait que les sons aient leur propre vie et existent dans une mer de silence - eh bien, c'est de l'improvisation. Il y a improvisation à partir du moment où l'on produit un son, parce que quelqu'un est présent en le produisant, ce que l'on peut, par analogie, étendre à une plus large texture. Si l'on est vraiment dans l'instant, exactement - et comme chaque moment passe, on ne pense pas à ce que I'on a déjà fait, mais on demeure présent -, alors on improvise ou on joue John Cage.

Car lorsque Cage dit «tout est possible», les gens oublient la partie conséquente de l'énoncé « ... en partant de rien », où le mot « rien » signifie « dépourvu d'habitudes et d'intentions".

J.R. : Comment est-ce possible pour un interprète d'être dans le bon état d'esprit pour jouer la musique de Cage, "en partant de rien »?
(2) Malcolm Goldstein a dirigé le Concert for Piano and Orchestra avec Aria à Francfort en 1991, avec le Hessischer Rundfunk Ensemble für Neue Musik. 
M.G. : Avant tout, il ne s'agit pas de techniques. J'ai dû résoudre cela pour interpréter Theatre Piece. Quand on fait quelque chose, on fait quelque chose. On ne pense ni à l'effet que cela peut produire, ni aux personnes qui pourraient aimer ou haiir, ni à rien. On ne réfléchit qu'à ce que l'on fait. Alors, tout change.

\section{J.R. : Est-ce possible ?}

M.G. : Nous le faisons à chaque minute de notre vie ! Par contre, en devenant interprètes, cela se modifie. Eł c'est là que Cage est important, par exemple avec Theatre Piece, de la même façon qu'avec le Concert for Piano and Orchestra. Cela fait intégralement partie de sa manière de travailler : rien ne va nulle part. Cage nous situe dans le temps - un espace de temps - dans lequel des choses arrivent, où rien n'est véritablement dit, néanmoins nous recevons quantité d'informations. Si quelqu'un monte sur la scène et se dit « je dois faire ceci pour donner l'impression que c'est "artistique" ", c'est autre chose et l'auditoire le perçoit. Lorsqu'on accomplit simplement une action, c'est alors ce que les spectateurs voient. C'est pour eux une expérience merveilleuse et très unique. Quand on pense, on n'agit pas. Si on agit, on agit ; si on réfléchit, on réfléchit. Cela est pour moi un aspect fondamental de l'improvisation.

\section{J.R. : C'est très pragmatique !}

M.G. : Exactement. Et de certains points de vue, c'est très difficile. En fait, ça peut être très simple ou très ardu. Au départ, quelqu'un veut réfléchir et comprendre.

"Comment est-ce que je produis un son?"

On lui dit : "Vous faites un son."

- Non, je veux dire : Comment dois-je vraiment produire un son?"

Les mots «vraiment " ou " devoir » sont révélateurs... ils proviennent d'un système de hiérarchie des valeurs.

"Non, vous faites simplement un son".

Dans les années soixante, j'ai travaillé avec des danseurs du Judson Dance Theatre. II s'agissait de personnes dont plusieurs avaient étudié avec Merce Cunningham. Et voir quelqu'un tout simplement marcher, voir des gens courir autour d'une pièce et nommer cela de la danse !... Issu d'une tout autre sphère d'activités, i'ai acquiescé : «Oui, c'est magnifique! » C'est à ce moment que i'ai commencé à opter pour l'improvisation. Le travail concret avec la danse a donc défait la rigidité de ma pensée. De la même façon, je crois que Merce et John ont institué un terreau propice à la croissance.

J.R. : Mais pourquoi monter sur une scène pour accomplir des gestes qui sont si naturels? 
M.G. : Parce que la clarté dont j'ai parlé au sujet de l'interprétation n'est pas courante. Je crois que nous recevons beaucoup de la nature. Un arbre est un arbre ; il est, tout simplement. Un arbre ne dit pas « aimez-moi » ou « haïssezmoi ». Pourtant, on en reçoit quelque chose de fantastique. Puis, nous revenons dans notre monde, celui des humains. Pour nous, avoir cette clarté - et c'est ici que le zen entre en ligne de compte - et l'amener dans un espace de représentation est une expérience tout à fait particulière pour l'interprète et pour les spectateurs. C'est une autre forme d'esthétique; c'est un partage. Ce n'est pas que je ne me préoccupe pas des auditeurs, mais je ne me soucie pas de leurs réactions. C'est comme ouvrir une porte et permettre aux gens d'entrer; par leurs perceptions ils se déplacent vers une direction ou une autre, et je ne suis qu'une énergie par laquelle ils participent ou s'éloignent... Ce n'est pas quelque chose de linéaire qui dit précisément ou qui se propulse résolument à l'extérieur; c'est davantage une dilatation qu'un élément qui vous frappe.

En improvisation, les gens apprennent à se faire confiance. Dans Theatre Piece, il faut faire beaucoup de choix. Cage sous-entend: "Je vous fais confiance. " Mais il s'agit davantage d'un lien de confiance entre les interprètes et le matériau, encore plus le matériau que le compositeur. Si on l'approche avec intelligence ou sympathie, il y a alors de multiples possibilités.

Les auditeurs aussi apprennent la confiance en soi. C'est un phénomène où leur perception, leur façon d'écouter la pièce constitue la pièce. Comme dans Theatre Piece hier soir, il n'y a pas d' « œuvre "; c'est un processus. C'est trente minutes d'activités qui interagissent parce que les actions arrivent dans le même espace-temps. La définition de l'art et celle des auditeurs s'établit alors différemment. Ce n'est pas une hiérarchie entre ceux qui savent et ceux qui ne savent pas; ou sur ce qu'est la vérité. La musique expérimentale conduit à ce genre de questionnement, ce qui a amené Cage à s'intéresser à l'anarchie. Sa musique comporte une dynamique sociale qui est en fait politique, en présentant un cadre anarchique, où les activités et les individus sont respectés pour eux-mêmes plutôt qu'à l'intérieur d'un ordre hiérarchique.

J.R. : Pourtant, il y a toujours des indications temporelles dans Theatre Piece, il y a une méthode à laquelle vous devez vous soumettre. Cette méthode est pour moi une contrainte, même si elle peut permettre la multiplicité ou la clarté.

M.G. : Il n'y a rien de tel que d'être totalement libre. Nous pouvons être libérés de quelque chose ou libre de faire quelque chose. Cela est tout à fait différent. Penser que quelqu'un effectue une activité qui ne comporte pas de limitations est impossible. Theatre Piece équivaut à un processus semblable de délimitation des possibilités. Nous pouvons rassembler huit personnes et dire : faisons une pièce d'une durée moyenne où chacun d'entre nous choisira certaines activités, que nous ferons courtes; disons qu'elles ne seront pas toutes de la même longueur et que nous essayerons de les varier ; mélangeons-les, 
parfois, pour former une sorte de collage ; réservons des moments où nous ne ferons rien... Je suis maintenant en train d'élaborer la pièce de Cage et plus i'emploie de mots pour définir les activités, plus je circonscris l'œuvre.

C'est la question de savoir jusqu'où l'on définit quelque chose. Dans les pièces de Cage ou d'autres compositeurs qui incorporent l'improvisation, il y a plusieurs brèches, des portes ouvertes qui permettent aux gens d'ajouter des éléments, ce qui est très spécial dans une situation de représentation ; ou, dans Theatre Piece, pour choisir quoi faire. Dans le processus de la composition, Cage pose des questions qui définissent ensuite la pièce et les définitions sont des limitations. Ce ne sont pas des contraintes, mais plutôt des clarifications au sujet de la convergence des éventualités.

La responsabilité de John, en tant que compositeur, était d'être aussi clair que possible, pour que les interprètes comprennent le temps, les limitations, les éléments à explorer. C'est la différence entre une musique complètement écrite et ces "compositions - improvisations structurées ». Cela conduit à un rapport totalement différent entre Cage, son interprète et l'auditoire. Je me suis trouvé dans des situations où les auditeurs questionnaient: "Comment ose-til se nommer le compositeur? " En fait, il est le compositeur, car lui seul est responsable de cela.

J.R. : Vous croyez donc que l'œuvre est unique et ouverte, mais Cage est toujours le compositeur comme n'importe quel compositeur. Ce n'est pas une contradiction pour vous?

M.G. : Non. La beauté de l'approche anarchique est que nous apprécions les différences entre les gens, en prenant pour acquis que ce sont des aspects que nous avons en commun. Cela est intégré à la musique. Et je pense que ça effraie des gens, car il y a une perte de contrôle. C'est ce qui est implicitement dangereux à propos de Cage, et il est compréhensible que les gens qui veulent garder le contrôle aient peur. D'un autre côté, s'ils peuvent prendre plaisir à cette perte de maîtrise, ils en deviendront peut-être très heureux! Bref, il s'agit $d^{\prime}$ accomplir une action ou de produire un son sans intention. II est implicite dans tous les écrits de Cage sur l'anarchie, de même que dans toute son œuvre, qu'il énonce un état de fait au sujet de ce qui n'est pas un état de fait.

Traduit de l'anglais par Johanne Rivest. 